JOURNAL OF THE

CHUNGCHEONG MATHEMATICAL SOCIETY

Volume 27, No. 2, May 2014

http://dx.doi.org/10.14403/jcms.2014.27.2.219

\title{
AN OPTIMAL CONSUMPTION AND INVESTMENT PROBLEM WITH LABOR INCOME AND REGIME SWITCHING
}

\author{
YONG HYun SHIN*
}

\begin{abstract}
I use the dynamic programming approach to study the optimal consumption and investment problem with regimeswitching and constant labor income. I derive the optimal solutions in closed-form with constant absolute risk aversion (CARA) utility and constant disutility.
\end{abstract}

\section{Introduction}

Following the seminal works of Merton [3, 4], the field of continuoustime portfolio selection is one of the most important areas in mathematical finance. Also recently regime-switching technique is widely used in mathematical finance (see $[1,7,5,6]$ ).

In this work I study the optimal consumption and investment problem with two-state regime-switching and constant labor income under the dynamic programming framework based on Karatzas et al. [2]. I use the constant absolute risk aversion (CARA) utility function and constant disutility to derive the optimal solutions in closed-form.

\section{The financial market}

On a proper probability space $(\Omega, \mathcal{F}, \mathbb{P})$, a standard Brownian motion $B_{t}$ and a continuous-time two-state Markov chain $\epsilon_{t}$ are defined and it is assumed that they are independent. The filtration $\left\{\mathcal{F}_{t}\right\}_{t \geq 0}$ is generated by both the Brownian motion $B_{t}$ and the Markov chain $\epsilon_{t}$.

Received January 10, 2014; Accepted April 07, 2014.

2010 Mathematics Subject Classification: Primary 91G10.

Key words and phrases: regime-switching, labor income, CARA utility, portfolio selection. 
In the financial market, it is assumed that only two assets are traded: One is a riskless asset with constant interest rate $r>0$ and the other is a risky asset (or stock). It is also assumed that there are two regime states, 1,2 in the market and regime $i$ switches into regime $j$ at the first jump time of an independent Poisson process with intensity $\lambda_{i}$, for $i, j \in\{1,2\}$. In regime $i \in\{1,2\}$, the risky asset price process follows $d S_{t} / S_{t}=\mu_{i} d t+\sigma_{i} d B_{t}$. The market price of risk is defined by $\theta_{i}:=$ $\left(\mu_{i}-r\right) / \sigma_{i}, i=1,2$. Let $\pi_{t}$ be the $\mathcal{F}_{t}$-progressively measurable portfolio process at time $t$ and $c_{t}$ be the nonnegative $\mathcal{F}_{t}$-progressively measurable consumption rate process at time $t$. I assume that the portfolio process $\pi_{t}$ and the consumption rate process $c_{t}$ satisfy the following conditions:

$$
\int_{0}^{t} \pi_{s}^{2} d s<\infty \text { and } \int_{0}^{t} c_{s} d s<\infty \text {, for all } t \geq 0 \text {, almost surely (a.s.). }
$$

In regime $i \in\{1,2\}$, the agent receives constant labor income $y_{i}>0$. The agent's wealth process $X_{t}$ at time $t$ follows

$$
d X_{t}=\left[r X_{t}+\pi_{t}\left(\mu_{i}-r\right)-c_{t}+y_{i}\right] d t+\sigma_{i} \pi_{t} d B_{t}, \quad X_{0}=x>-\frac{y_{i}}{r}, i=1,2 .
$$

\section{The optimization problem}

The agent's expected utility maximization problem with CARA utility $u(c):=-e^{-\gamma c} / \gamma$ is given by

$$
V_{i}(x)=\sup _{(c, \pi) \in \mathcal{A}(x)} \mathbb{E}\left[\int_{0}^{\tau_{i}} e^{-\rho t}\left(-\frac{e^{-\gamma c_{t}}}{\gamma}-l\right) d t+e^{-\rho \tau_{i}} V_{j}\left(X_{\tau_{i}}\right)\right]
$$

where $\tau_{i}$ is the first jump time from $i$-th state to $j$-th state, $\rho>0$ is a subjective discount factor, $\gamma>0$ is the coefficient of absolute risk aversion, $l>0$ is constant disutility because of labor, and $\mathcal{A}(x)$ is an admissible class of pairs $(c, \pi)$ at $x$, where $i, j \in\{1,2\}$ and $i \neq j$. It is assumed that the following inequality always holds without further comments:

Assumption 3.1.

$$
\rho-r+\frac{\theta_{i}^{2}}{2}>0, i \in\{1,2\} .
$$

Assumption 3.2. It is assumed that the value function $V_{i}(x)$ for this optimization problem (3.1) is an increasing function, that is,

$$
V_{i}^{\prime}(x)>0 \text {, for } i=1,2 .
$$


In fact, $V_{i}^{\prime}(x)>0$ (see (3.11)).

My main results are given in the next theorem.

Theorem 3.3. The value function for this optimization problem (3.1) is given by

$$
V_{i}(x)=-\frac{1}{r \gamma} M_{i} e^{-\gamma\left(r x+y_{i}\right)}-\frac{l}{\rho}, i=1,2,
$$

where $\left(M_{1}, M_{2}\right)$ is the unique pair solution of the system of algebraic equations

$$
\left(\rho-r+\lambda_{i}+\frac{1}{2} \theta_{i}^{2}\right) e^{-\gamma y_{i}} M_{i}+r e^{-\gamma y_{i}} M_{i} \log M_{i}-\lambda_{i} e^{-\gamma y_{j}} M_{j}=0,
$$

for $i, j \in\{1,2\}$ and $i \neq j$. And the optimal policies $\left(c_{i}^{*}, \pi_{i}^{*}\right)$ for this optimization problem (3.1) are given by

$$
c_{i}^{*}=r x+y_{i}-\frac{1}{\gamma} \log M_{i} \text { and } \pi_{i}^{*}=\frac{\theta_{i}}{\sigma_{i} r \gamma}, i=1,2 .
$$

Proof. From the expected utility optimization problem (3.1), I derive the coupled Bellman equations

$$
\begin{gathered}
\max _{\left(c_{i}, \pi_{i}\right)}\left[\left\{r x+\pi_{i}\left(\mu_{i}-r\right)-c_{i}+y_{i}\right\} V_{i}^{\prime}(x)+\frac{1}{2} \sigma_{i}^{2} \pi_{i}^{2} V_{i}^{\prime \prime}(x)\right. \\
\left.-\left(\rho+\lambda_{i}\right) V_{i}(x)+\lambda_{i} V_{j}(x)-\frac{e^{-\gamma c_{i}}}{\gamma}-l\right]=0,
\end{gathered}
$$

where $i, j \in\{1,2\}$ and $i \neq j$. The first-order conditions (FOCs) for the Bellman equations (3.2) give

$$
c_{i}^{*}=-\frac{1}{\gamma} \log \left\{V_{i}^{\prime}(x)\right\} \quad \text { and } \quad \pi_{i}^{*}=-\frac{\theta_{i} V_{i}^{\prime}(x)}{\sigma_{i} V_{i}^{\prime \prime}(x)}, i=1,2 .
$$

Plugging the FOCs (3.3) into the equations (3.2), then I obtain

$$
\begin{gathered}
r x V_{i}^{\prime}(x)+y_{i} V_{i}^{\prime}(x)-\frac{1}{2} \theta_{i}^{2} \frac{\left\{V_{i}^{\prime}(x)\right\}^{2}}{V_{i}^{\prime \prime}(x)}+\frac{1}{\gamma} V_{i}^{\prime}(x) \log \left\{V_{i}^{\prime}(x)\right\} \\
-\left(\rho+\lambda_{i}\right) V_{i}(x)+\lambda_{i} V_{j}(x)-\frac{1}{\gamma} V_{i}^{\prime}(x)-l=0,
\end{gathered}
$$

where $i, j \in\{1,2\}$ and $i \neq j$. Now it is assumed that the optimal consumption $c_{i}^{*}=C_{i}(x), i=1,2$, is a function of wealth $x$. And let $X_{i}(\cdot), i=1,2$, be the inverse function of $C_{i}(\cdot), i=1,2$, that is, $X_{i}(\cdot)=C_{i}^{-1}(\cdot), i=1,2$. Then the FOCs (3.3) give

$$
V_{i}^{\prime}(x)=e^{-\gamma C_{i}(x)} \quad \text { and } \quad V_{i}^{\prime \prime}(x)=-\frac{\gamma e^{-\gamma C_{i}(x)}}{X_{i}^{\prime}\left(c_{i}\right)}, i=1,2 .
$$


Substituting (3.5) into the equations (3.4), then I have

$$
\begin{aligned}
& r X_{i}\left(c_{i}\right) e^{-\gamma c_{i}}+y_{i} e^{-\gamma c_{i}}+\frac{1}{2 \gamma} \theta_{i}^{2} X_{i}^{\prime}\left(c_{i}\right) e^{-\gamma c_{i}}-c_{i} e^{-\gamma c_{i}} \\
& \quad-\left(\rho+\lambda_{i}\right) V_{i}\left(X_{i}\left(c_{i}\right)\right)+\lambda_{i} V_{j}\left(X_{i}\left(c_{i}\right)\right)-\frac{1}{\gamma} e^{-\gamma c_{i}}-l=0,
\end{aligned}
$$

where $i, j \in\{1,2\}$ and $i \neq j$. Taking derivative of the equations (3.6) with respect to $c_{i}$ yields

$$
\begin{aligned}
& r X_{i}^{\prime}\left(c_{i}\right) e^{-\gamma c_{i}}-r \gamma X_{i}\left(c_{i}\right) e^{-\gamma c_{i}}-\gamma y_{i} e^{-\gamma c_{i}}+\frac{1}{2 \gamma} \theta_{i}^{2} X_{i}^{\prime \prime}\left(c_{i}\right) e^{-\gamma c_{i}} \\
& -\frac{1}{2} \theta_{i}^{2} X_{i}^{\prime}\left(c_{i}\right) e^{-\gamma c_{i}}+\gamma c_{i} e^{-\gamma c_{i}}-\left(\rho+\lambda_{i}\right) X_{i}^{\prime}\left(c_{i}\right) e^{-\gamma c_{i}}+\lambda_{i} X_{i}^{\prime}\left(c_{i}\right) e^{-\gamma c_{j}}=0,
\end{aligned}
$$

where $i, j \in\{1,2\}$ and $i \neq j$. Thus I derive the coupled second order ordinary differential equations (ODEs)

$$
\begin{aligned}
& \frac{1}{2 \gamma} \theta_{i}^{2} X_{i}^{\prime \prime}\left(c_{i}\right)-\left(\rho-r+\lambda_{i}+\frac{1}{2} \theta_{i}^{2}\right) X_{i}^{\prime}\left(c_{i}\right)-r \gamma X_{i}\left(c_{i}\right) \\
& \quad+\gamma c_{i}-\gamma y_{i}+\lambda_{i} X_{i}^{\prime}\left(c_{i}\right) e^{-\gamma\left(c_{j}-c_{i}\right)}=0,
\end{aligned}
$$

where $i, j \in\{1,2\}$ and $i \neq j$. If I conjecture the solution $X_{i}\left(c_{i}\right)$ to the coupled ODEs (3.7) of the form

$$
X_{i}\left(c_{i}\right)=\frac{c_{i}-y_{i}}{r}+\frac{1}{r \gamma} \log M_{i} \quad \text { and } \quad c_{i}=r x+y_{i}-\frac{1}{\gamma} \log M_{i}, i=1,2 \text {, }
$$

for some constant $M_{i}>0$, then $X_{i}^{\prime}\left(c_{i}\right)=1 / r$ and $X_{i}^{\prime \prime}\left(c_{i}\right)=0, i=1,2$. The equations (3.8) yield

$$
c_{j}-c_{i}=y_{j}-y_{i}+\frac{1}{\gamma} \log \frac{M_{i}}{M_{j}},
$$

where $i, j \in\{1,2\}$ and $i \neq j$. Thus the coupled ODEs (3.7) can be reduced into the system of algebraic equations

$$
\left(\rho-r+\lambda_{i}+\frac{1}{2} \theta_{i}^{2}\right) e^{-\gamma y_{i}} M_{i}+r e^{-\gamma y_{i}} M_{i} \log M_{i}-\lambda_{i} e^{-\gamma y_{j}} M_{j}=0,
$$

where $i, j \in\{1,2\}$ and $i \neq j$. Let $N_{i}:=e^{-\gamma y_{i}} M_{i}>0$, then I obtain

$$
\left(\rho-r+\lambda_{i}+\frac{1}{2} \theta_{i}^{2}+r \gamma y_{i}\right) N_{i}+r N_{i} \log N_{i}-\lambda_{i} N_{j}=0,
$$

where $i, j \in\{1,2\}$ and $i \neq j$.

Now I want to show that there exists a unique pair solution $\left(M_{1}, M_{2}\right)$ to the system of algebraic equations (3.9). Thus it is enough to show that 
there exists a unique pair solution $\left(N_{1}, N_{2}\right)$ to the system of algebraic equations (3.10). The proof is very similar to the proof of Theorem 3.2 in Shin [6]. Without loss of generality, I may assume that $\theta_{i}<\theta_{j}$. If I define

$$
N_{j}=f\left(N_{i}\right):=\frac{1}{\lambda_{i}}\left(\rho-r+\lambda_{i}+\frac{1}{2} \theta_{i}^{2}+r \gamma y_{i}\right) N_{i}+\frac{r}{\lambda_{i}} N_{i} \log N_{i}>0,
$$

for $N_{i}>0$, and

$$
f_{1}\left(N_{i}\right):=\frac{f\left(N_{i}\right)}{N_{i}}=\frac{1}{\lambda_{i}}\left(\rho-r+\lambda_{i}+\frac{1}{2} \theta_{i}^{2}+r \gamma y_{i}\right)+\frac{r}{\lambda_{i}} \log N_{i}>0,
$$

then $f_{1}^{\prime}\left(N_{i}\right)=r /\left(\lambda_{i} N_{i}\right)>0$, that is, $f_{1}(\cdot)$ is increasing. Now I define the constants $\bar{x}$ and $\underline{x}$ with $\bar{x}>\underline{x}$ as follows:

$$
\bar{x}:=e^{-\frac{1}{r}\left(\rho-r+\frac{1}{2} \theta_{i}^{2}\right)}<1 \quad \text { and } \quad \underline{x}:=e^{-\frac{1}{r}\left(\rho-r+\lambda_{i}+\frac{1}{2} \theta_{i}^{2}+r \gamma y_{i}\right)}<1,
$$

where the inequalities are obtained from Assumption 3.1. Then $f_{1}(\bar{x})=$ $1+r \gamma y_{i} / \lambda_{i}, f_{1}(\underline{x})=0$. Thus I have $N_{i}>\underline{x}$ since $f_{1}\left(N_{i}\right)>0$ and $f_{1}(\cdot)$ is increasing.

Now I define

$$
\begin{aligned}
g\left(N_{i}\right):= & \left(\rho-r+\lambda_{j}+\frac{1}{2} \theta_{j}^{2}+r \gamma y_{j}\right) N_{i} f_{1}\left(N_{i}\right) \\
& +r N_{i} f_{1}\left(N_{i}\right) \log \left\{N_{i} f_{1}\left(N_{i}\right)\right\}-\lambda_{j} N_{i},
\end{aligned}
$$

and

$$
\begin{aligned}
g_{1}\left(N_{i}\right): & =\frac{g\left(N_{i}\right)}{N_{i}} \\
& =\left(\rho-r+\lambda_{j}+\frac{1}{2} \theta_{j}^{2}+r \gamma y_{j}\right) f_{1}\left(N_{i}\right)+r f_{1}\left(N_{i}\right) \log \left\{N_{i} f_{1}\left(N_{i}\right)\right\}-\lambda_{j} .
\end{aligned}
$$

It can be checked that

$g_{1}(\bar{x})=\left\{\frac{1}{2}\left(\theta_{j}^{2}-\theta_{i}^{2}\right)+r \gamma y_{j}+r \log \left(1+\frac{r \gamma y_{i}}{\lambda_{i}}\right)\right\}\left(1+\frac{r \gamma y_{i}}{\lambda_{i}}\right)+\frac{r \gamma \lambda_{j} y_{i}}{\lambda_{i}}>0$.

Since, by l'Hospital's rule,

$$
\begin{aligned}
& \lim _{N_{i} \rightarrow \underline{x}+} f_{1}\left(N_{i}\right) \log \left\{N_{i} f_{1}\left(N_{i}\right)\right\} \\
& =\lim _{N_{i} \rightarrow \underline{x}+} \frac{\log \left\{N_{i} f_{1}\left(N_{i}\right)\right\}}{1 / f_{1}\left(N_{i}\right)}=\lim _{N_{i} \rightarrow \underline{x}+} \frac{f_{1}\left(N_{i}\right)\left(f_{1}\left(N_{i}\right)+N_{i} f_{1}^{\prime}\left(N_{i}\right)\right)}{-N_{i} f_{1}^{\prime}\left(N_{i}\right)}=0,
\end{aligned}
$$

$\lim _{N_{i} \rightarrow \underline{x}+} g_{1}\left(N_{i}\right)=-\lambda_{j}<0$. Thus, by intermediate value theorem, there exists $\bar{N}>0$ such that $g_{1}(\bar{N})=0$ and $\underline{x}<\bar{N}<\bar{x}$. Taking derivative of 
$g_{1}\left(N_{i}\right)$ gives

$$
\begin{aligned}
g_{1}^{\prime}\left(N_{i}\right) & =\left(\rho+\lambda_{j}+\frac{1}{2} \theta_{j}^{2}+r \gamma y_{j}\right) f_{1}^{\prime}\left(N_{i}\right)+r f_{1}^{\prime}\left(N_{i}\right) \log \left\{N_{i} f_{1}\left(N_{i}\right)\right\}+r \frac{f_{1}\left(N_{i}\right)}{N_{i}} \\
& =\frac{r}{\lambda_{i} N_{i}} h\left(N_{i}\right),
\end{aligned}
$$

where

$h\left(N_{i}\right):=\left(2 \rho-r+\lambda_{i}+\lambda_{j}+\frac{\theta_{i}^{2}+\theta_{j}^{2}}{2}+r \gamma\left(y_{i}+y_{j}\right)\right)+r \log \left\{N_{i}^{2} f_{1}\left(N_{i}\right)\right\}$.

Taking derivative of $h\left(N_{i}\right)$ implies

$$
h^{\prime}\left(N_{i}\right)=\frac{2 r}{N_{i}}+r \frac{f_{1}^{\prime}\left(N_{i}\right)}{f_{1}\left(N_{i}\right)}>0 .
$$

Thus $h(\cdot)$ is increasing. Also note that $\lim _{N_{i} \rightarrow \underline{x}+} h\left(N_{i}\right)=-\infty$ and

$$
h(\bar{x})=r+\lambda_{i}+\lambda_{j}+\frac{\theta_{j}^{2}-\theta_{i}^{2}}{2}+r \gamma\left(y_{i}+y_{j}\right)+r \log \left(1+\frac{r \gamma y_{i}}{\lambda_{i}}\right)>0 .
$$

Again, by intermediate value theorem, there exists a unique $x^{*}>0$ such that $h\left(x^{*}\right)=0$ and $\underline{x}<x^{*}<\bar{x}$. Thus $h\left(N_{i}\right)<0$ for $\left(\underline{x}, x^{*}\right)$ and $h\left(N_{i}\right)>0$ for $\left(x^{*}, \infty\right)$ since $h(\cdot)$ is increasing. This means $g_{1}^{\prime}\left(N_{i}\right)<0$ for $\left(\underline{x}, x^{*}\right)$ and $g_{1}^{\prime}\left(N_{i}\right)>0$ for $\left(x^{*}, \infty\right)$. Thus $g_{1}\left(N_{i}\right)$ is decreasing and negative for $\left(\underline{x}, x^{*}\right)$ and $g_{1}\left(N_{i}\right)$ is increasing for $\left(x^{*}, \infty\right)$. Therefore $\bar{N}$ with $x^{*}<\bar{N}<\bar{x}$ is the unique solution to $g_{1}\left(N_{i}\right)=0$, and this implies that I have the unique pair solution $\left(N_{1}, N_{2}\right)$ to (3.10). Therefore I obtain the unique pair solution $\left(M_{1}, M_{2}\right)$ to $(3.9)$.

Now substituting $c_{i}$ in (3.8) into (3.5) yields

$$
V_{i}^{\prime}(x)=M_{i} e^{-\gamma\left(r x+y_{i}\right)}>0 \quad \text { and } \quad V_{i}^{\prime \prime}(x)=-r \gamma M_{i} e^{-\gamma\left(r x+y_{i}\right)}<0, i=1,2 .
$$

Also substituting (3.11) into the FOCs (3.3) implies the optimal policies

$$
c_{i}^{*}=r x+y_{i}-\frac{1}{\gamma} \log M_{i} \text { and } \pi_{i}^{*}=\frac{\theta_{i}}{\sigma_{i} r \gamma}, i=1,2 .
$$

Therefore the Bellman equations (3.2) gives the value function

$$
V_{i}(x)=-\frac{1}{r \gamma} M_{i} e^{-\gamma\left(r x+y_{i}\right)}-\frac{l}{\rho} .
$$




\section{References}

[1] B. G. Jang, H. K. Koo, H. Liu, and M. Loewenstein, Liquidity Premia and Transaction Costs, J. Finance 62 (2007), 2329-2366.

[2] I. Karatzas, J. P. Lehoczky, S. P. Sethi, and S. E. Shreve, Explicit Solution of a General Consumption/Investment Problem, Math. Oper. Res. 11 (1986), 261-294.

[3] R. C. Merton, Lifetime Portfolio Selection under Uncertainty: the ContinuousTime Case, Rev. Econ. Stat. 51 (1969), 247-257.

[4] R. C. Merton, Optimum Consumption and Portfolio Rules in a ContinuousTime Model, J. Econ. Theory 3 (1971), 373-413.

[5] Y. H. Shin, Portfolio Selection with Regime-Switching: Dynamic Programming Approaches, J. Chungcheong Math. Soc. 25 (2012), 277-281.

[6] Y. H. Shin, Optimal Consumption and Investment Problem with RegimeSwitching and CARA Utility, J. Chungcheong Math. Soc. 26 (2013), 85-90.

[7] L. R. Sotomayor and A. Cadenillas, Explicit Solutions of ConsumptionInvestment Problems in Financial Markets with Regime Switching, Math. Finance 19 (2009), 251-279.

Department of Mathematics

Sookmyung Women's University

Seoul 140-742, Republic of Korea

E-mail: yhshin@sookmyung.ac.kr 